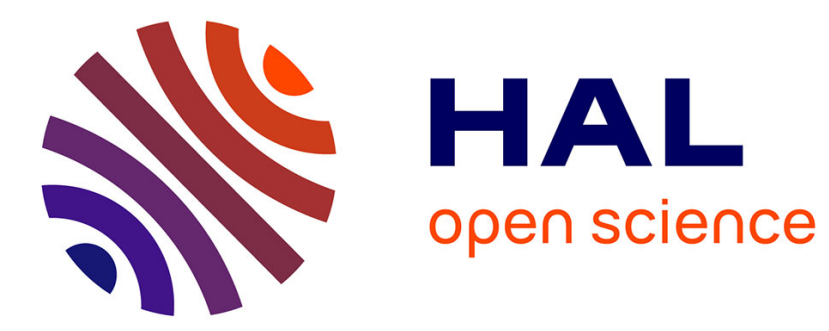

\title{
Scenography of immersive virtual musical instruments
}

\author{
Florent Berthaut, Victor Zappi, Dario Mazzanti
}

\section{To cite this version:}

Florent Berthaut, Victor Zappi, Dario Mazzanti. Scenography of immersive virtual musical instruments. VR Workshop: Sonic Interaction in Virtual Environments (SIVE), 2014 IEEE, Mar 2014, Minneapolis, United States. 10.1109/SIVE.2014.7006285 . hal-01222454

\section{HAL Id: hal-01222454 \\ https://hal.science/hal-01222454}

Submitted on 30 Oct 2015

HAL is a multi-disciplinary open access archive for the deposit and dissemination of scientific research documents, whether they are published or not. The documents may come from teaching and research institutions in France or abroad, or from public or private research centers.
L'archive ouverte pluridisciplinaire HAL, est destinée au dépôt et à la diffusion de documents scientifiques de niveau recherche, publiés ou non, émanant des établissements d'enseignement et de recherche français ou étrangers, des laboratoires publics ou privés.

\section{다(1)(2)}

Distributed under a Creative Commons Attribution - ShareAlike| 4.0 International 


\section{Scenography of Immersive Virtual Musical Instruments}

\author{
Florent Berthaut* \\ Department of Computer Science, \\ University of Bristol
}

\author{
Victor Zappi ${ }^{\dagger}$ \\ School of Engineering and Computer Science \\ Queen Mary University of London
}

\author{
Dario Mazzanti \\ Department of Advanced Robotics, \\ Istituto Italiano di Tecnologia
}
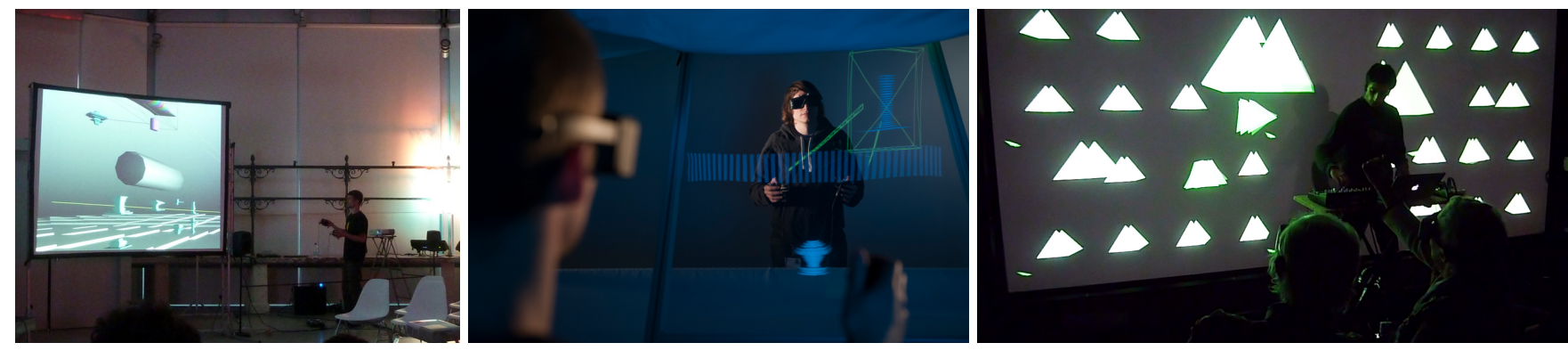

Figure 1: IVMI performances with various stage setups: Angled screens, Semi-transparent screen, and Single screen

\begin{abstract}
Immersive Virtual Musical Instruments (IVMIs) can be considered as the meeting between Music Technology and Virtual Reality. Being both musical instruments and elements of Virtual Environments, IVMIs require a transversal approach from their designers, in particular when the final aim is to play them in front of an audience, as part of a scenography. In this paper, we combine the main constraints of musical performances and Virtual Reality applications into a set of dimensions, meant to extensively describe IVMIs stage setups. A number of existing stage setups are then classified using these dimensions, explaining how they were used to showcase live virtual performances and discussing their scenographic level.
\end{abstract}

Keywords: Immersive virtual musical instruments, scenography, audience's experience, virtual reality.

\begin{abstract}
Index Terms: - Human-centered computing Virtual reality - Human-centered computing Scenario-based design - Human-centered computing Heuristic evaluations - Human-centered computing HCI theory, concepts and models
\end{abstract}

\section{INTRODUCTION}

\subsection{Context}

In theatre, cinema and television, scenography relates to the study and the development of visual, experiential and spatial composition of performance. One of the main aims of this practice is to fully immerse the audience in the production, eliciting emotional and rational engagement [14].

This concept is surely familiar to New Media artists who have designed immersive Virtual Reality (VR) installations. Although technologically different, similar scenographic solutions are used in VR-based art to grant strong involvement of the audience, who is also the user of the interactive 3D application. However, when

\footnotetext{
*e-mail: florent@hitmuri.net

†e-mail: victor.zappi@qmul.ac.uk

‡e-mail: dario.mazzanti@iit.it
}

moving towards the context of VR performances, the concept of "user" and "audience" are no more overlapped. Performers are on stage, they interact with 3D objects inside a Virtual Environment (VE), in front of a separated audience. In this case, who is supposed to be the main target of the scenography? And is it possible to equally address both performers and spectators?

In these works, while the VE is designed to maximize performer's perception and control capabilities, spectators' virtual experience is not generally taken into account. As a result, often only performers are considered "users", as opposed to the spectators, who are barely confined outside of the VE. The concept of scenography is still present, but looses its audience-oriented connotation.

This issue is particularly important in the case of Immersive Virtual Musical Instruments (IVMI). These devices rely on the representation of sound processes and parameters as 3D entities in a VE and on fine 3D interaction techniques. In particular, their precise use always implies a certain level of immersion of the musicians, which can be granted by the use of a proper technological setup. However, the higher is the level of immersion provided by the stage setup (i.e., more refined instrument control), the more abstract and disconnected from the VE is likely to be the audience's experience.

The usage of a poor audience-oriented scenography may obviously also affect the spectators' strict musical experience, which combines sonic material and the perception of the musicianinstrument relationship. When not immersed in the VE, spectators lack important information about the instrument and performers' gestures, limiting musical engagement to only intellectual and cognitive levels [7]. This issue is common to most electronic instruments, but, in the case of IVMIs, it is not solely inherent to the nature of the instrument's metaphor, rather it can partly derive from the chosen technological setup.

So far, several examples of IVMIs have been presented in literature, some featuring metaphors relying on high levels of musician's immersion, like [21], [12], or [1], others, for their nature, more oriented towards a clear display of the musician-instrument relationship, for example [16], [15] or [23]. Although they can all be used (some have been) for public performances, no studies have been done on how to setup a proper scenography for these instruments to engage the audience.

The aim of this work is to help instrument makers and performers, but also designers of VR applications in other fields, to create better scenographies for public exhibitions of their own systems. 


\subsection{Contribution}

Our contribution is two-fold :

- First, we propose a set of dimensions to evaluate the stage setups of IVMIs and virtual musical performances, based on the analysis of the most common constraints in musical performances and VR.

- Then, we use these dimensions to describe, classify and compare some example stage setups, specifically evaluating features related to the concept of scenography.

\section{Constraints of Musical performances and Vir- TUAL REALITY APPLICATIONS}

\subsection{Musical Performances}

In this section, we define several constraints which need to be taken into account when designing a musical performance.

\subsubsection{Understanding the Instrument}

Differently from concerts performed with acoustic or electric instruments, electronic music concerts often feature new instruments, such as hardware controllers or laptops, which may distract or confuse the audience. Indeed, the sound of acoustic instruments is easy to guess from their categories, such as wind instruments, strings or percussions, and physical properties, such as size or material. Moreover, their sound and the mappings between performed gestures and sonic results are fixed and characterise the instrument. The behaviour of some non-acoustic instruments such as electric guitars or keyboards is less constrained but equally well known, mainly because they are used by several musicians.

On the other hand, new instruments are harder to understand for several reasons. First of all, they may use any sound synthesis technique and any mapping between musician's gestures and sound parameters, as described in [8] or [22]. There is also no consistency between the sound produced by new instruments and their appearance, i.e. hardware controller shape or graphical user interface. Moreover, the behaviour of such instruments may change over time, even during a single performance. They are also less known, as they are used by fewer musicians, or even only their developer. Therefore, it is important to make sure that the audience can understand how the instrument is working. Graphical interfaces may ease this by displaying components, structures and behaviour of instruments, especially when multiple processes [9] are used. The behaviour of new instruments can be amplified for the benefit of the audience, using 3D graphical visualizations. For example, in Rouages [2] 3D shapes are used to represent the components of new instruments and their sound generation, in order to enrich spectators' experience.

When designing 3D instruments, solutions such as immersion using large screens, stereoscopy and head-tracking may provide a better perception of the graphical components, and therefore a better understanding of the instrument.

\subsubsection{Understanding Musician's Gestures}

Besides understanding how an instrument works, the audience should also be able to perceive the relation between musician's gestures and their result. Such connection deeply affects the liveness of a musician's performance with an instrument, and consequently the audience's experience [13]. This can be even more crucial for instruments using several sound processes, as it may be hard to differentiate between processes controlled by musicians and autonomous processes. In performances with laptops for example, soundscapes tend to be rich and it can be almost impossible to perceive musicians gestures, as they consist of small hands and fingers movements, often hidden behind the screen. As suggested by Cascone [5], the audience may feel frustrated under these circumstances, because they have no way to understand if musicians are really doing something or only reading their e-mails.

Musicians' gestures and their consequences are defined as manipulations and effects by Reeves et al. [17]. They qualify public interfaces, such as new musical instruments, in relation to how much manipulations and effects are hidden from the audience. According to this taxonomy, expressive instruments reveal and even amplify both manipulations and effects, positively affecting audience's experience.

In the case of IVMIs, gestures and the instrument belong to two different worlds, the physical world and the VE. For this reason, simply showing the effects of musician's gesture on the instrument could be difficult. Some solutions should be considered to integrate the instrument in the physical space in a way that the VE blends perfectly with the real environment.

\subsubsection{Communication Between Musicians and the Audience}

Another important constraint is communication. Indeed, Bongers [3] points out that musical performance involves non-verbal communication between musicians and the audience. It is fundamental that in one way, musicians can see reactions of audience, so that they can for example adapt their playing in the case of an improvisation, or simply so that they get better feeling of the ambiance. In the other way, spectators should be able to see, in addition to their gestures, expression and looks of musicians, especially in the context of a collective performance.

\subsection{VR Constraints}

In this section, we define what constraints influence the user experience in VR. In line with the aim of the study, first we consider a classic user-centered paradigm, then an audience-performer paradigm.

\subsubsection{Immersion}

Immersion is a key constraint in VR. The term refers to the description of the technology used to arouse in the user the psychological sense of being in the VE [19]. This specific state of consciousness, experienced by the user and targeted in all immersive user-centered VR applications, has been defined as presence. In an optimal scenario, when a user feels "present" in a virtual world, he/she acts as if the environment were real, physically and emotionally engaged in the application.

Moving to a VR audience-performer scenario, immersion deeply affects both the audience's and the musician's experience. An immersive performance acts on the audience's feeling of presence within the VE used on stage. As a consequence, the virtual instrument and all its 3D graphical components can be perceived, to a certain extent, as "real". This aspect highly impacts the strength of virtual interaction, as seen from the eyes of the spectators; the gestures performed by the musician to play the instrument become more and more meaningful and communicative, as the instrument, from virtual, becomes real. On the other hand, when interaction takes place in a far and complete different environment (i.e. when the audience's feeling of presence is low or almost absent), performer's poses and gestures are perceived as obscure and even awkward [11]. Moreover, even if the VE does not include interactive $3 \mathrm{D}$ objects, but it is capable of physically reaching and surrounding the audience, the emotional engagement of the spectators in the performance is sensibly enhanced [23]

Likewise, from the musician's point of view, the perception of the VE and of the IVMI as real entities has remarkable consequences on the whole performance. In this case, immersion grants a more natural interaction with the instrument, resulting into a more expressive output, and influences control and confidence, which are strongly connected to the concept of virtuosity [10]. 


\subsubsection{Reality-Virtuality Continuity}

In immersive VR, presence is achieved through a combination of techniques and technologies, that are functional elements of the global system; some examples are multimodal feedbacks, detailed graphics, high framerate and large tracking area. As discussed in [6], the effects of all these different elements are highly interconnected with one another, while the absence or the misuse of most of them produces breaks in presence [4].

As discussed later in this work, most of the stage setups we will analyse are characterized by a combination of real and virtual objects interacting together (in first instance the virtual instrument and the musician). For this reason, it is worth focusing on the subset of immersive elements which grant a proper perceptive connection between reality and virtuality. We can call this subset RealityVirtuality Continuity (RVC), which includes (but it is not limited to) tracking precision and real world noise filtering.

Although it can not assure a complete immersion, considering a classic user-centered paradigm the effect of RVC is twofold. First of all, from the user's perspective real physical objects integrated into the scene are visually aligned with the VE, also when the point of view changes. Moreover, all the unwanted stimuli (i.e. noise) coming from the real world surrounding the VR setup (e.g. people talking, touch or feel of VR equipment) are properly filtered, minimizing breaks of presence due to external reasons [20].

According to an audience-performer paradigm, RVC has to be considered as an even stronger constraint, although it's fulfillment requires more complex operations. As discussed by Sanchez and Slater [18], immersion is generally grounded on the possibility of interacting with the environment. But in most cases, spectators have not an active role in the performance; they do not interact with the scenography, they rather watch the musician playing the virtual instrument. In such an exocentric scenario, the total absence of interactive capabilities increases the effect of the perceived RVC onto the global level of immersivity of the VE on stage. In other words, action is replaced by a correct and robust perception of a real entity (i.e. the musician) interacting with virtual objects. As pointed out in [23] this positively influences the audience's feeling of presence during VR performances.

From the performer's point of view, RVC is a fundamental constraint for the implementation of consistent interaction algorithms, necessary to make possible a natural usage of the virtual instrument.

Unfortunately, the immersive technologies used in virtual performances are generally incapable of preserving RVC from both sides. Often, the chosen setups penalize the musician's experience in favor of the audience's, or vice versa. These aspects and their dependencies with the scenography will be addressed in details in the next sections.

\section{SCENOGRAPHY DimENSIONS}

In this section we define the six dimensions for the scenography of IVMIs and their impact on the constraints related to musical performances and VR that we defined in section 2.

\subsection{Musician Immersion}

Musician immersion relates to the immersion of the musician(s) in the VE which comprises the instrument, as defined in the previous section. It ranges from no immersion (e.g. monoscopic flat displays with fixed perspective) to complete immersion (e.g. flat stereoscopic displays with dynamic perspective or volumetric displays). It impacts mostly the interaction of the musician(s) with the instrument.

\subsection{Audience Visibility}

Audience visibility corresponds to the level of perception of the audience by the musician(s). In other words, it defines how well the musician(s) can perceive the audience. This dimension ranges from no perception at all to directly facing and seeing the physical audience. It impacts the communication between the musician(s) and the audience, as defined in section 2.1.3.

\subsection{Audience immersion}

Similarly to musician immersion, this dimension describes how well the audience perceives the VE and therefore the instrument. In addition to the values defined for the musician immersion, this dimension also includes conflicts between the musician and audience immersion, for example when they share the same screen but the perspective is computed for the musician. Therefore the fact of having a separate projection or screen improves the audience immersion. This dimension impacts mostly the understanding of the instrument by the audience.

\subsection{Musician Visibility}

Musician visibility corresponds to the perception of the musician(s) by the audience. It ranges from not seeing the musician at all to seeing him facing the audience. It impacts both the communication and the understanding of musicians' gestures by spectators.

\subsection{Gestures Continuity}

Gestures continuity relates to how the musical gestures performed by musicians in the physical space are connected to the graphic feedback of the instrument's metaphor, as perceived by the audience. It ranges from no continuity, for example when musician(s) can not be seen, to full continuity when the gestures are perfectly aligned with the components of the $3 \mathrm{D}$ interaction techniques. This dimension impacts the understanding of the instrument and of musicians' gestures. In turn, it depends on the audience immersion and on musician visibility.

\subsection{From Virtual to Physical}

The from virtual to physical dimension corresponds to how the virtual and physical spaces are merged. At one end, musician(s) and instrument are both rendered on a screen which acts as a window into the VE, completely detached from the real world. At the other end, the instrument is displayed as if it was in the physical space, ensuring perceptual consistency of the merged virtual and physical elements. Between these two ends, the instrument can be for example part of a VE contained in a virtual volume within the physical space. This dimension impacts the gestures continuity, which is easier to ensure if everything is integrated in the VE, and communication.

\section{Classification of Existing Stage Setups}

In this section we use the six IVMI scenography dimensions to analyse five different stage setups. In particular, we consider how these setups were used to stage 3 specific virtual performances; this allows for practical observations on scenography and possible improvements of each solution. A visual representation of the analysis is given on Figure 2 in the form a dimension space, that provides a quick overview of each setup's properties. In particular, the top part axes gives an indication on the level of communication. The right part axes show how well the audience perceive the instrument and musical gestures.

\subsection{Head Mounted Display and Screen}

We start this series of examples with one of the first and simplest stage setups ever designed to showcase a virtual performance in front of an audience. It consists of a Head Mounted Display (HMD) used in combination with a projected screen.

The most famous virtual performance capitalizing on this stage setup is probably The Sound of One Hand [11], which took place in 1993. On stage, Jaron Lanier was in turn using different IVMIs sparse all over a VE. Sounds and notes were generated by hand 


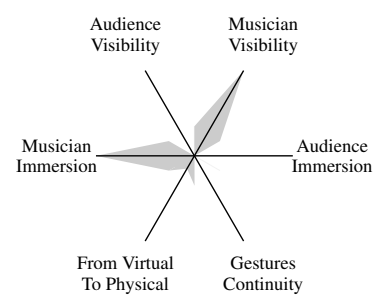

HMD and detached screen

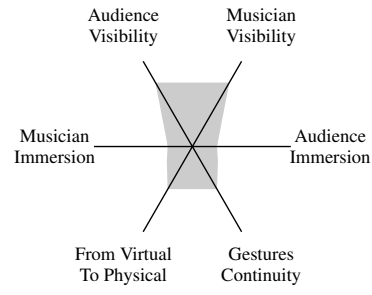

Angled screens

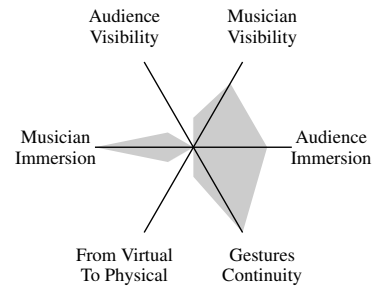

3D reconstruction

Figure 2: Dimension Spaces for the analysed stage setups.

movements, as they were transmitted to the instruments using a dataglove; this same device was used to move inside the VE using a point-fly technique. On stage, placed aside of the musician, a screen was used to display a $2 \mathrm{D}$ projection of his view point.

The primary dimension of this stage setup is musician immersion; the use of the HMD allowed for the musician to perceive a consistent world all around him and to have access to fine 3D controls. However, the use of the HMD totally prevented the audience to be seen by the musician, hence resulting into a total absence of audience visibility. Conversely, musician visibility is quite high: he played on the stage, right in front of the audience, but he was also free to move and rotate, yet partially hiding his gestures. Gestures continuity is almost absent, since the VE and the musician were perceived by the audience as two completely separated elements, the former projected onto a screen, the latter on the physical stage. For the same reason, the from virtual to physical dimension is strongly biased towards virtuality. Furthermore, the screen projection was $2 \mathrm{D}$ and it displayed the musician's point of view, resulting into an extremely low level of audience immersion.

The scenographic level of this setup is quite poor, since it mainly focuses on the musician and his virtual interaction. However, showing spectators a $3 \mathrm{D}$ projection aligned with the physical position of the musician on stage would remarkably enhance the audience's experience, providing immersion and increasing gestures continuity.

\subsection{Single Screen}

This setup is based on a single screen, positioned on stage behind the musician(s), as shown in Figure 4. The screen is used to display stereoscopic projections to the audience, comparably to what

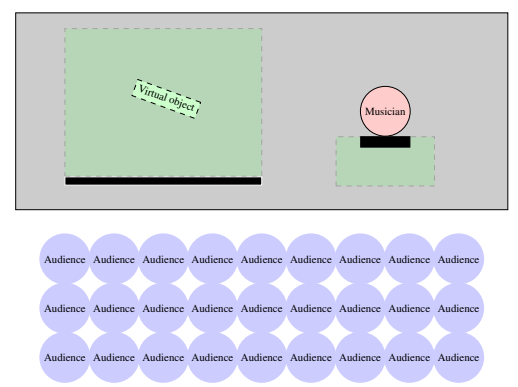

Figure 3: Scheme for the single screen stage setup. The musician wears an hmd and the audience sees his point of view on the screen placed on the stage.

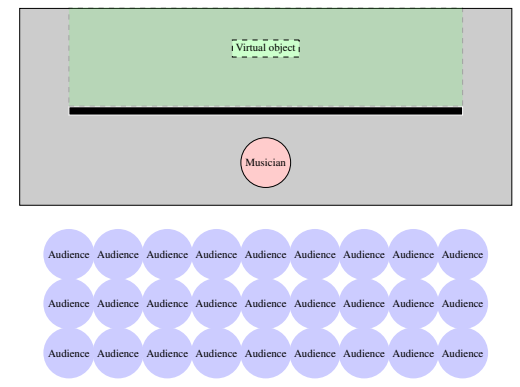

Figure 4: Scheme for the single screen stage setup. The musician performs in front of the screen.

happens in movie theatres.

The single screen setup was used to stage the Virtual_Real performance [23], in which an electronic musician played using both standard hardware controllers and non-immersive virtual instruments. A VE was displayed onto the screen, providing choreographic feedback through 3D visual. Virtual instruments were represented by 3D objects, which could be moved, touched or morphed by the performer in order to control audio effects. Such manipulation relied on optical motion capture of one of the musician's hands.

This stage setup is strongly focused towards providing an intense audience experience. The stereoscopic imagery merging with the real stage strengthens both audience immersion and musician visibility. From virtual to physical dimension is moved towards the physical end, since the VE and its instruments are perceived by the audience as coherently superimposed with the physical stage. These features and the use of motion capture, allow musician's manipulation of the virtual instruments to be consistently aligned with the audience's point of view, generating a high gestures continuity. If we consider the performer's point of view, it is easy to see how musician immersion is absent, since the performer faces the audience and not the screen. On the other hand, since the musician directly sees the audience audience visibility is maximum.

Scenographies based on single screen setups can create strong involvement in the audience, capitalizing on virtual choreographies and non-verbal communication with the performers. However, such an extremely audience-centric setup makes impossible the usage of IMVIs based on complex 3D interactions. Slight setup modifications could generate a dual experience, in which the screen projection is dedicated to the performer, completely changing the scenographic outcome. The audience would no longer enjoy the perfect virtual/real environments consistency, while musicians would be immersed in the instrument, allowing for fine audio control. 


\subsection{Angled Screens}

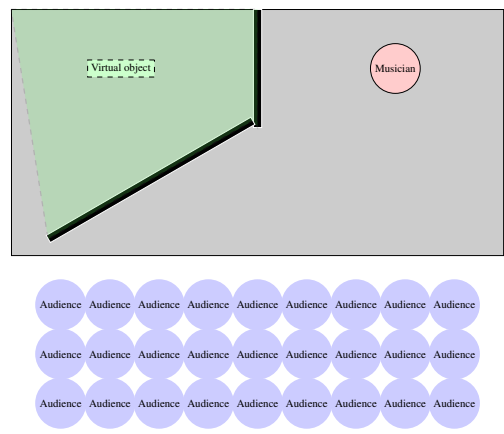

Figure 5: Scheme for the angled screen stage setup, based on the juxtaposition of two screens.

In this example, two screens are juxtaposed facing different directions, so as to completely enclose a part of the stage. One screen is facing the performer, sideways, the other one is angled, dedicated to the audience (Figure 5). In this way both audience and performers perceive the VE as "contained" inside of this virtual volume.

The IVMI analysed using this particular setup is Drile [1]. The instrument itself relies on the manipulation of 3D audiovisual objects that are associated with the nodes of live-looping trees. Handheld devices with pressure sensors are used to reach these musical objects by means of a virtual ray technique and to excite/modulate them, creating loops. Since only remote virtual interaction was implied, the setup could be successfully used to enclose the whole instrument within the stage area in between the two screens, as depicted on Figure 1. Both the projections were monoscopic.

The audience immersion is relatively low, since, although they were assigned a dedicated view on the VE, their projection was monoscopic. Similarly, the musician immersion is low due to the lack of stereoscopic projection and head-tracking. The audience visibility and musician visibility are quite high, since musician and spectators directly saw each other from the side. The gestures continuity is medium low. The virtual ray indicates what the musician was manipulating but the musician operated on the instrument standing one or more meters away from the screen, breaking the continuity between his hands and the virtual rays. For the virtual to physical dimension, this setup was at half-way, because the instrument was in a virtual volume enclosed in the physical space.

This setup and its scenographic level could be vastly improved by simply introducing stereoscopic projections for both the musician and the audience. However, as for the previous setup, only a single sweet spot (defined as the center of the seat area) would be shared by spectators; however, since the stage and the seat area are generally some meters far from each other, this would not dramatically affect the audience's experience. Furthermore, propagating the virtual ray between the musician and the virtual volume through, for example, projection mapping would specifically increase the scenographic value of this stage setup.

\subsection{Semi-transparent Screen}

This setup relies on the Pepper's ghost effect, obtained using an acrylic transparent screen. This screen is placed between the musician and the audience and rotated of 45 degrees on the horizontal axis, the lower part being on the audience side. Above and below it two projection screens are set. The projection screen placed above is reflected on the audience side of the semi-transparent screen while the one below is reflected on the musician side. Therefore a different rendering can be displayed on each side without interference, and the screen remains see-through.

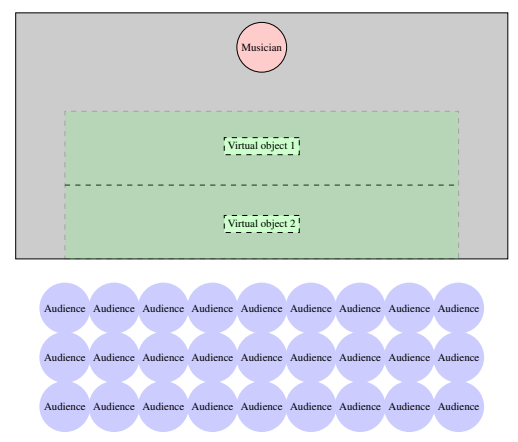

Figure 6: Scheme for the stage setup based on the semi-transparent screen. People at both sides can perceive 3D projections without any interference.

We will use another performance featuring the Drile instrument to analyse the setup. As depicted on Figure 1, during the performance, the two renderings on the opposite sides of the tilted screen showed the same 3D audiovisual objects that composed Drile, but from two opposite perspectives (one from the front, the other from the back). Doing so, the instrument appeared to be placed right in between the musician and the audience. The rest of the instrument's equipment remained the same.

The audience visibility and musician visibility are maximized since they face each other through the semi-transparent screen. The audience immersion is high. The VE was rendered through a stereoscopic passive projector. However, again only one sweet spot was shared among the audience. The musician immersion is very high since the projection on his side was also stereoscopic and since his head was tracked to adjust the rendering to his point of view. The gestures continuity is medium/high. The semi-transparency of the setup allowed the audience to perceive continuity between musicians gestures and virtual rays; nonetheless, whenever the tilted screen is not completely flat, distortion can occur and affect the perceived alignment. This setup was at the physical end of the virtual to physical dimension, the instrument being completely integrated in the physical space thanks to the Pepper's ghost effect.

Scenographies based on this setup can be very effective, also preserving the performers' experience. It is important to notice that the complexity of the equipment used may in turn introduce other issues, more related to the technical preparation of the stage (e.g. distortions due to an imprecise framing of the tilted screen).

\subsection{D Reconstruction}

In this specific setup, the stage is divided into two parts, one visible to the audience and another one completely concealed (the backstage). As depicted in Figure 7, musicians perform exclusively in the backstage and are recorded by one or more cameras; the visible part of the stage hosts only a screen, where both the VE and avatars of the musicians are rendered.

Recently, again Drile has been performed using this stage setup. The instrument was prepared in the backstage and included all the equipment necessary for it to be played. Additionally, a depth camera was used for a real-time $3 \mathrm{D}$ reconstruction of the musician. This avatar was then displayed on the stage screen, facing the audience. Doing so, the VE was perceived by the spectators as if positioned between them and the performer.

Since the setup included stereoscopic equipment for both the IMVI and the visible stage, both musician immersion and audience immersion are particularly high. Both VE and musician were rendered on the screen, so the stage was totally virtual (from virtual to physical) and this allowed for the perception of high ges- 

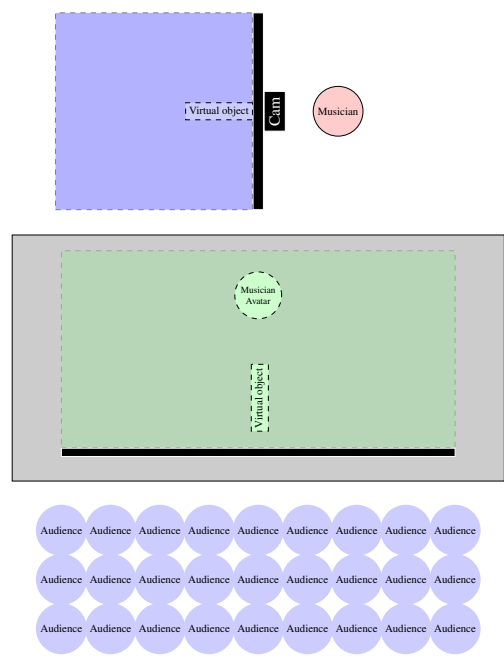

Figure 7: Scheme for the stage setup based on 3D reconstruction of the performer. musician and audience are in two separate part of the stage.

tures continuity. On the other hand, the separation between stage and backstage determined a complete absence of audience visibility for the musicians and a limited level of musician visibility, since bounded to the resolution of the used camera.

The scenography built on this setup is strong and it does not limit the musician's freedom of action in any ways. However, it notably lacks many important characteristics typical of live performances, resembling more a recorded performance. The add of a dual system to reconstruct the spectators could be used to display them back to the musician, closing the visual communication loop.

\section{Conclusion}

In this paper we presented a set of dimensions that allow for the description of the scenography of performances with immersive virtual musical instruments. They rely on the analysis of the constraints of musical performances and virtual reality setups. We show that these dimensions might serve as a classification tool for existing performances but also as a design tool to help improve existing stage setups and build new ones. We hope that other researchers, instrument makers and musicians will rely on them for their own performances and help us refining them. Finally, we think that these dimensions could be adapted to non-musical immersive performances such as dance or theater.

\section{REFERENCES}

[1] F. Berthaut, M. Desainte-Catherine, and M. Hachet. Drile : an immersive environment for hierarchical live-looping. In Proceedings of New Interfaces for Musical Expression (NIME10), pages 192-197, Sydney, Australia, 2010.

[2] F. Berthaut, T. Marshall, Mark, S. Subramanian, and M. Hachet. Rouages: Revealing the Mechanisms of Digital Musical Instruments to the Audience. In Proceedings of the Conference on New Interfaces for Musical Expression, page 6 pages, Daejeon, Corée, République De, 2013.

[3] B. Bongers. Exploring novel ways of interaction in musical performance. In $C \& C$ '99: Proceedings of the $3 r d$ conference on Creativity \& cognition, pages 76-81, New York, NY, USA, 1999. ACM.

[4] A. Brogni, M. Slater, and A. Steed. More breaks less presence. In Proceedings of Presence 2003: The 6th Annual International Workshop on Presence, 2003.

[5] K. Cascone. Laptop music - counterfeiting aura in the age of infinite reproduction. Parachute, issue 107, 2002.
[6] D. Cho, J. Park, G. J. Kim, S. Hong, S. Han, and S. Lee. The dichotomy of presence elements: The where and what. In $V R$, pages 273-274. IEEE Computer Society, 2003.

[7] M. Gurevich and A. Cavan Fyans. Digital musical interactions: Performer-system relationships and their perception by spectators. Organised Sound, 16:166-175, 62011.

[8] A. Hunt and R. Kirk. Mapping strategies for musical performance. Trends in Gestural Control of Music, pages 231-258, 2000.

[9] S. Jordà. Interactive music systems for everyone: exploring visual feedback as a way for creating more intuitive, efficient and learnable instruments. In Proceedings of the Stockholm Music Acoustics Conference (SMAC03), Stockholm, Sweden, 2003.

[10] S. Jordà. Digital instruments and players part ii: Diversity, freedom and control. In International Computer Music Conference, 2004

[11] J. Lanier. The sound of one hand. Whole Earth Review, 1993.

[12] T. Mäki-Patola, J. Laitinen, A. Kanerva, and T. Takala. Experiments with virtual reality instruments. In Proceedings of the 2005 International Conference on New Interfaces for Musical Expression (NIME05), Vancouver, BC, Canada, pages 11-16, 2005.

[13] M. Marshall, P. Bennett, M. Fraser, and S. Subramanian. Emotional response as a measure of liveness in new musical instrument performance. In CHI 2012 Workshop on Exploring HCI Relationship with Liveness, May 2012.

[14] J. McKinney and P. Butterworth. The Cambridge Introduction to Scenography. Cambridge Introductions to Literature. Cambridge University Press, December 2009.

[15] A. G. Mulder. Design of virtual three-dimensional instruments for sound control. PhD thesis, Simon Fraser University, Canada, 1998.

[16] J. Oliver and S. Pickles. Fijuu2: a game-based audio-visual performance and composition engine. In NIME' '07: Proceedings of the 7th international conference on New interfaces for musical expression, pages 430-430, New York, NY, USA, 2007. ACM.

[17] S. Reeves, S. Benford, C. O'Malley, and M. Fraser. Designing the spectator experience. In Proceedings of the SIGCHI conference on Human factors in computing systems, CHI '05, pages 741-750, New York, NY, USA, 2005. ACM.

[18] M. V. Sanchez-Vives and M. Slater. From presence to consciousness through virtual reality. Nature Reviews Neuroscience, 6:332-339, Apr. 2005.

[19] M. Slater, V. Linakis, M. Usoh, R. Kooper, and G. Street. Immersion, presence, and performance in virtual environments: An experiment with tri-dimensional chess. In ACM Virtual Reality Software and Technology (VRST 1996), page 163-172, 1996.

[20] M. Slater and A. Steed. A virtual presence counter. Presence, 9(5):413-434, 2000

[21] L. Valbom and A. Marcos. Wave: Sound and music in an immersive environment. Comput. Graph., 29:871-881, December 2005.

[22] M. M. Wanderley and P. Depalle. Gestural control of sound synthesis. In Proceedings of the IEEE, pages 632-644, 2004.

[23] V. Zappi, D. Mazzanti, A. Brogni, and D. Caldwell. Design and evaluation of a hybrid reality performance. In NIME'11: Proceedings of the 2011 conference on New interfaces for musical expression, pages 355-360, Oslo, Norway, 2011. 